\title{
ESC CardioMed
}

Thomas F. Lüscher ${ }^{1}$, John A. Camm², Gerald Maurer ${ }^{3}$, Patrick Serruys ${ }^{4}$

${ }^{1}$ Research, Education and Development, Royal Brompton and Harefield Hospital Trust and Imperial College, London, United Kingdom

${ }^{2}$ Molecular and Clinical Sciences Research Institute, Cardiology Clinical Acade ic Group,

St. George's University of London, London, United Kingdom

${ }^{3}$ Division of Cardiology, Department of Internal Medicine II, Medical University of Vienna, Wien, Austria ${ }^{4}$ Department of Cardiology, Imperial College of London, United Kingdom

Reprinted with permission from: Eur Heart J. 2018; 19: 959-961

The new ESC Textbook of Cardiovascular Medicine, ESC CardioMed, is a continuosly updating electronic database (Fig. 1).

\section{Education - the mission of the ESC}

The education of trainees, postgraduate and established cardiologists alike, and other health care proffesionals has always been a critical component of the mission of the European Society of Cardiology. Besides the most successful congresses of the main Society as well as its associations, the growing ESC Journal Family and also the ESC Textbook of Cardiovascular Medicine have been important products of this successful strategy.

\section{The ESC Textbook of Cardiovascular Medicine}

The first edition of the ESC Textbook of Cardiovascular Medicine was published in print in 2009 by Blackwell, with John Camm, Thomas F. Lüscher and Patrick Serruys as editors. Following the success of the first edition, the second edition appeared in 2011, this time published by Oxford University Press. Although available from the outset in a basic digital format, after the launch of the second edition the editors of the textbook felt that in today's modern world a more dynamic electronic version of a cardiology textbook with a sophisticated search system, many illustrations, and movies should be developed.



Figure 1. ESC CardioMed screenshot.

\section{ESC CardioMed}

Thus, in 2016 the ESC Board of the European Society of Cardiology decided to launch a third edition of the textbook, this time in a completely different format. The editors, now also including Gerald Maurer (Fig. 2), decided to enlarge the scope of the textbook to include many of the so far uncovered topics within the growing field of cardiovascular medicine such as embryology, catheter-based structural interventions, psycho-

Address for correspondence: Thomas F. Lüscher, MD, FRCP, Professor of Cardiology Imperial College, Director of Research, Education and Development, Royal Brompton and Harefield Hospitals, Sydney Street, London SW3 6NP, United Kingdom, e-mail: cardio@tomluescher.ch 


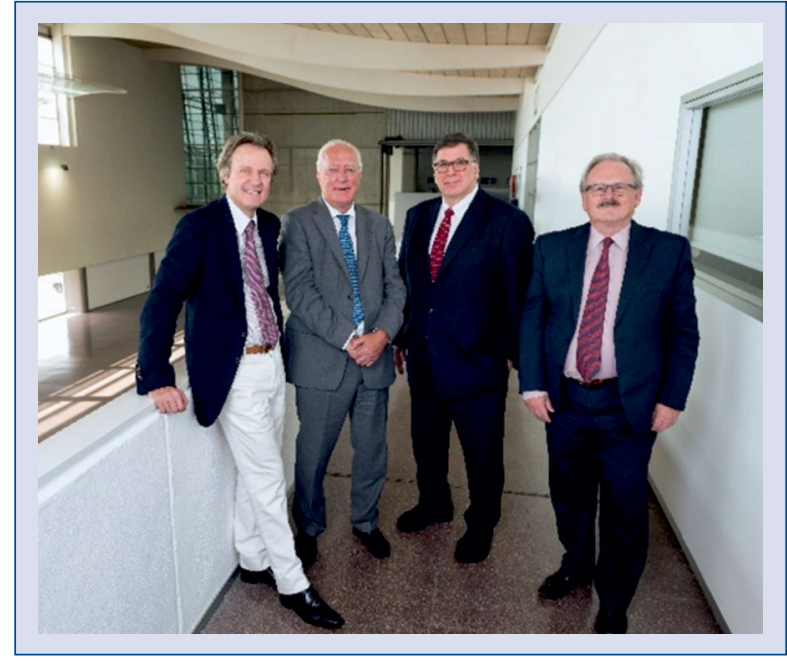

Figure 2. ESC CardioMed editors (from left to right): Thomas F. Lüscher, John A. Camm, Gerald Maurer, Patrick W. Serruys.

logical factors, ethnicity, interpretation of trials, and personalized medicine, to name but a few.

As such, the concept of a huge primarily electronic textbook was born with four main editors, 63 section co-ordinators, and more than 1000-chapter authors. As one can imagine, such a large project took more time than anticipated to develop, but the editors are now ready to proudly announce the publication of ESC CardioMed. Access to this huge electronic data base is available as a membership benefit for all Professional Members and Fellows of the European Society of Cardiology (Fig. 3). For others wishing to access the publication, annual subscriptions are available for individuals and institutions alike through the publisher.

In spring 2018 about one-third of the ESC CardioMed content became available online and now on the occasion of the Annual Congress of the European Society of Cardiology in Munich this August 2018, the majority of the chapters with a very few exceptions will be accessible online. The database is endowed with a sophisticated search system allowing rapid identification of the relevant parts of the book. Each of the chapters is illustrated, most with full colour figures and informative tables or videos of the most important clinical features. Indeed, particularly the imaging sections contain numerous videos of different cardiac conditions.

A novel aspect of the electronic ESC Textbook of Cardiovascular Medicine is first of all its name. It will be introduced under the name of ESC CardioMed to communicate its modern nature as a primarily comprehensive electronic database. Furthermore, and most importantly, it will be continuously updated three times a year, after each of the large cardiology congresses such as the Annual Congress of the European Society of Cardiology, the Scientific Sessions of the American Heart Association and the Annual Congress of the American College of Cardiology. Updates will primarily involve new trials affecting clinical practice or new imaging or interventional techniques highly relevant for the management of patients, as well as new insights into causes and mechanisms of cardiovascular disease of clinical interest. This will avoid the problem that most textbooks encounter, i.e. that they are outdated by the time they appear.

\section{The content}

The content of the textbook is divided into 63 sections that cover major aspects of cardiovascular disease (Table 1).

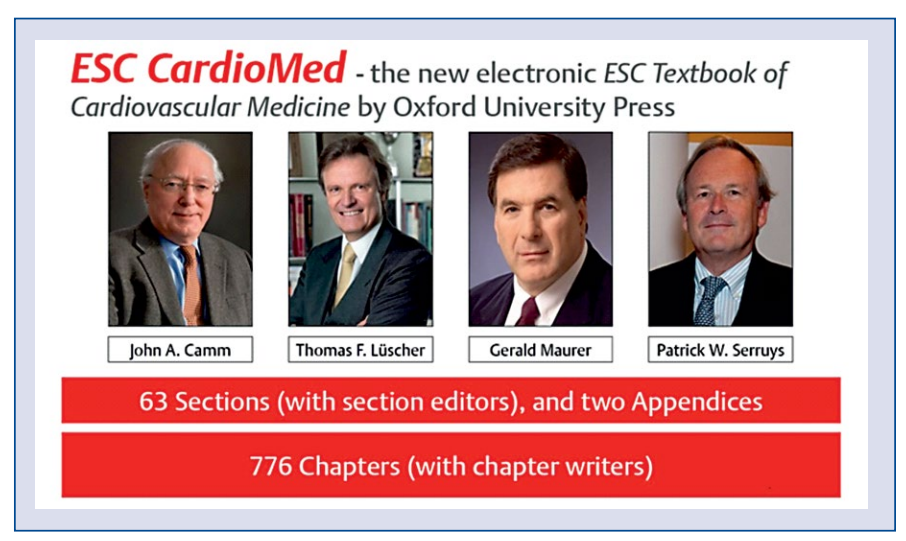

Figure 3. Structure of ESC CardioMed - the new electronic, continuously upgraded ESC Textbook of Cardiovascular Medicine edited by the European Society of Cardiology with Oxford University Press. 


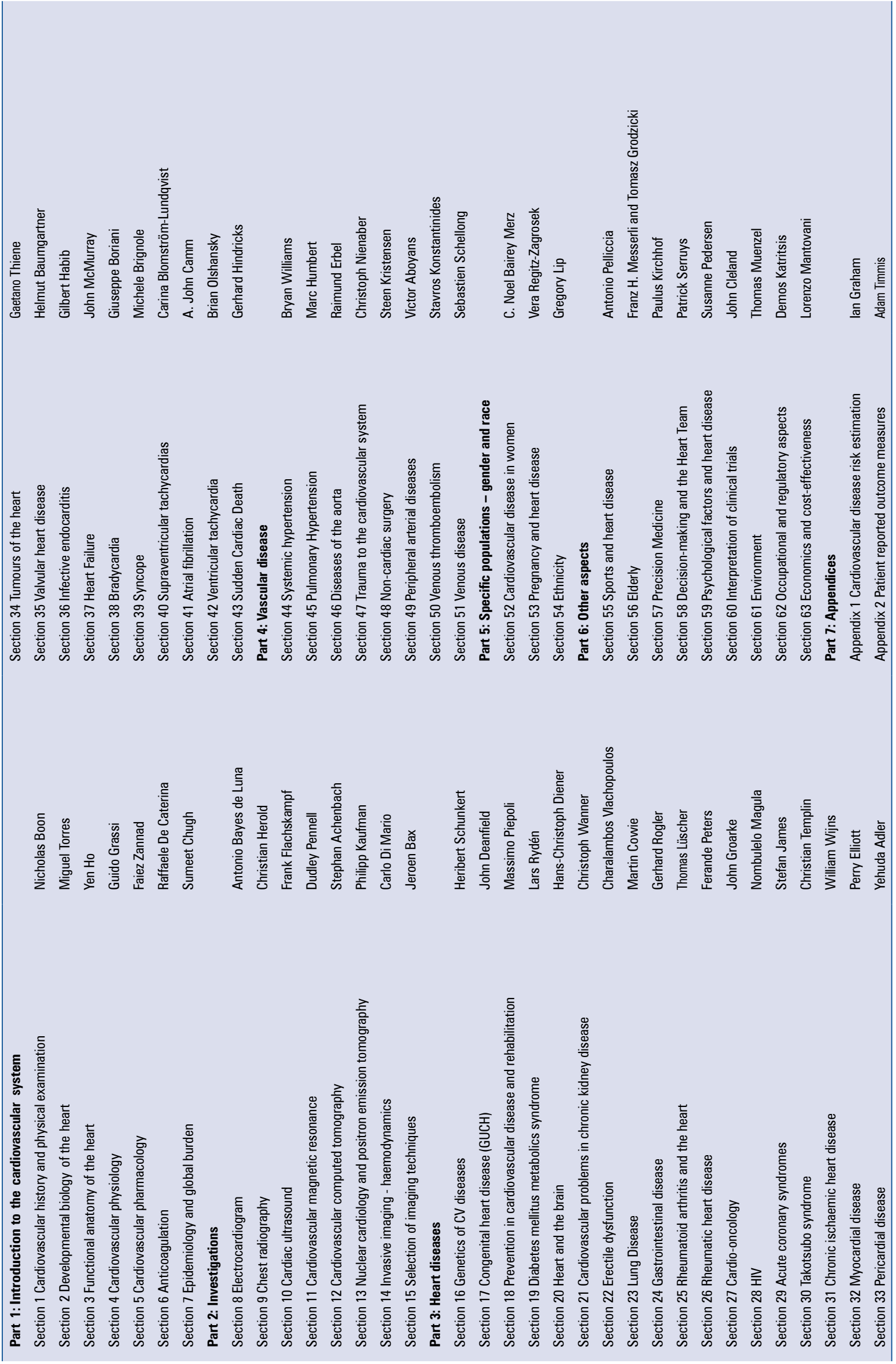




\section{Interaction with ESC Guidelines}

A further major new aspect of ESC CardioMed is its alignment with the ESC Guidelines. Many leaders in the field as well as the users of the ESC Guidelines have felt for some time that the documents were too long and difficult to read and it was difficult to find the main message. Therefore, the editors of ESC CardioMed came together with the ESC Guidelines Committee and its chairman Stephan Windecker and decided to gradually bring the two products together. To that end, it is envisaged that the ESC CardioMed will provide the background knowledge such as mechanisms of disease, epidemiology, and describe the trials in more detail, while the ESC Guidelines will focus primarily on the recommendations. Examples of this synergy are the direct links between ESC CardioMed and the most recent ESC Guidelines on Valvular Heart Disease and on Peripheral Arterial Disease published in the European Heart Journal. This concept will make the ESC Guidelines more readable and digestible and still provide the full information through the links with ESC CardioMed. This process will take some time to be completed but is currently ongoing as new ESC Guidelines are being developed.

A print version will be published as The ESC Textbook of Cardiovascular Medicine $3 e$ early in 2019 (Fig. 4).

The editors and the leaders of the European Society of Cardiology involved in this project do hope that ESC CardioMed will be frequently used

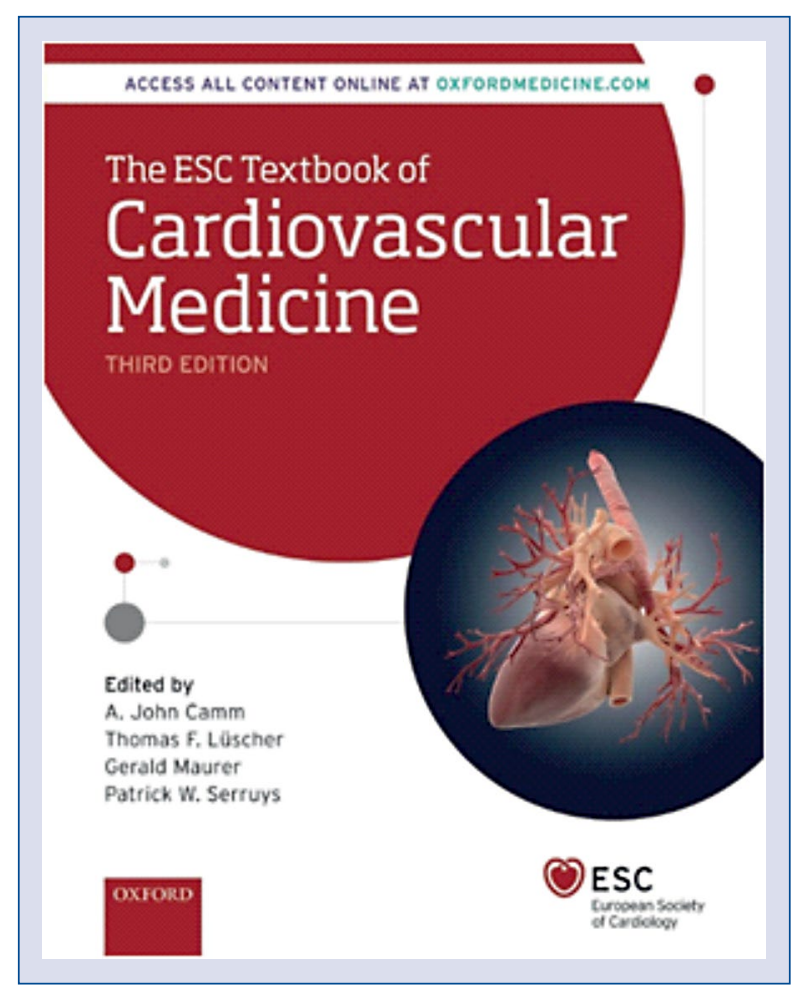

Figure 4. Front cover of the ESC Textbook of Cardiovascular Medicine, Oxford University Press, 2019.

by our cardiologists and other care professionals around the world and will turn out to be essential in their daily practice.

Conflict of interest: None declared 